\title{
COUPLING ENGINEERING KNOWLEDGE WITH PRODUCT DESIGN KNOWLEDGE BY THE MEANS OF MULTIPLE VIEW PRODUCT MODEL
}

\author{
Golnoosh Rasoulifar \\ PhD student \\ G-SCOP Laboratory \\ University of Grenoble, France \\ golnoosh.rasoulifar@g-scop.grenoble-inp.fr
}

\author{
Guy Prudhomme \\ Associate Professor \\ G-SCOP Laboratory \\ University of Grenoble, France \\ guy.prudhomme@g-scop.inpg.fr
}

\author{
Daniel Brissaud \\ Professor \\ G-SCOP Laboratory \\ University of Grenoble, France \\ daniel.brissaud@grenoble-inp.fr
}

\begin{abstract}
Product design involves a multitude of actors who have complementary knowledge and responsibility about the product under development.

Coupling together their views-points, particularly these of product designers and engineering designers in the upstream phases of the design, has the objectives to make the collaboration easier and to improve the product from both of their expertises.

The Product/Process multi-view model is a collaborative tool which supports engineering designers during product development processes. It allows the structuring and tracing of relative knowledge of engineering designers on the product. We make the assumption that Product/Process multi-view model could also be used to create relationships with the product designer's representation.

The paper deals with this product model and illustrates the possible connection between engineering knowledge and product design knowledge.
\end{abstract}

\section{INTRODUCTION}

We asked a product designer to describe one of his products (shown in figure 1). He said it is a red plastic bag that can be carried like a backpack. It has an oriented shape which gives the feeling of being aerodynamic and having a sportive appearance. The engineering designer described the same product not as a mono color bag but rather a combination of several parts with several colors, materials and shapes. This simple example shows the different ways that a product can be considered from -on one hand- a product designer point of view and on the other hand an engineering designer point of view, due to their expertness, knowledge and professional vision.

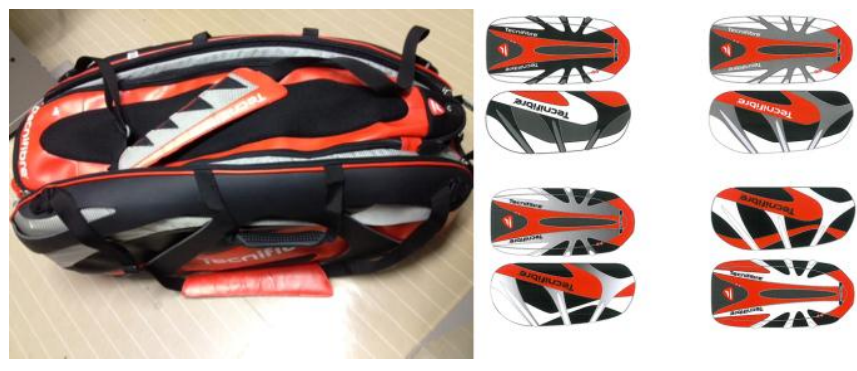

Figure 1, Tennis bag designed by Dièdre Design

Product design involves a multitude of actors who have complementary responsibilities about the product under development. In this article we distinguish two types of experts. The engineering designers are in charge of the product and process compliance into the technical measurable performances. The product designers are responsible for the overall product perception and aesthetics and they deal rather with the subjective meanings and sometimes non-measurable properties of the product. For the sector of brand products - which is our interest in this article- one challenge is that the products should differ from the competitors and express their own brand identity, so the product designer needs to focus on the identity of products which may come from the style, manufacture, technology, etc. Therefore better sharing of experts' knowledge between product designer and engineering designer results in better product design regarding to the brand requirements. 
Such problematic is part of the research subject of a national research project called SKIPPI (System for Kansei Image Product Process Innovation). The project aims to develop a software to support decision making in the upstream design phase which corresponds to task clarification phase according to systematic design approach of Pahl and Beitz (11).

Multi-view product model (see following sections for deepen explanations) is one possible way to represent a product in a design process. We decided to use Multi-view product model in our research because it makes possible to integrate different views carried out by the engineers in charge of the product development, even if the model is not been used for the same product sector as we study in our project (mobile phone, Tennis bag).

Considering the lexical nature of SKIPPI and its forecasted usage in preliminary phase of product design, when the product is not yet conceptualized, the compatibility of the hierarchical structure of Multi-view product model with SKIPPI requirements is problematic.

The aim of this article is twofold: to show if the Multi-view product model can support the engineering designers' view point for the brand product sector and if the model helps us to provide adequate information for the SKIPPI system.

In the primary sections the SKIPPI project is shortly described and the concepts of Kansei Engineering, Product models and more precisely Multi-View product model are presented. Afterwards, in the research method and experimentation sections, the principles of PP model are described. The contribution of the PP model to enrich the SKIPPI project is finally discussed in the results section.

\section{STATE OF THE ART SYSTEM FOR KANSEI IMAGE PRODUCT PROCESS INNOVATION}

SKIPPI is a three-year research project supported by National Research Agency (ANR) of France with both academic and industrial partners. The project concerns three research domains; the Kansei engineering, Product/Process integrated engineering and Artificial Intelligence. The objective of the SKIPPI system is to connect Kansei/Product/Process (KPP) (Figure2). The system is supposed to operate going from Kansei attributes (values, semantics, styles etc.) to the product and process parameters (components, color, manufacturing techniques etc.) and vice-versa from the PP parameters going to values. In other word, from a given word coming from emotions or perceptions or brand values (such as happy, beauty, young, comfortable) the system is supposed to generate ideas about using special material, or special shape, or special process or technology in product design. The system is also supposed to link the emphasized process or technologies (usually used by or new for the company) to generate product design ideas which raise special feeling or perception for the product user. For example, using a finishing process such as NCVM (Non Conductive Vacuum Metallization) on a component of product results in bright color for that component which gives a luxury feeling about the product. This additional information of the technical process could help the product designer in getting new ideas for designing a brand new product. Since the SKIPPI system is supposed to be used in the early stage of design process, when the ideas are not specified and detailed, the system deals only with words and underlines the relations between these words.

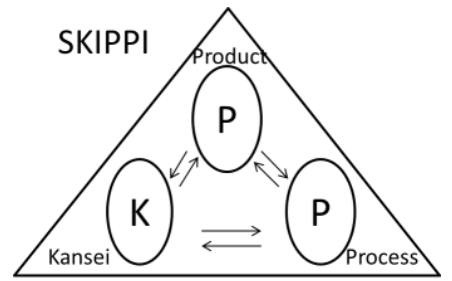

Figure 2: SKIPPI system aims to connect Kansei/Product/Process (KPP)

The project uses three lexical data bases. The first one concerns emotional and sensorial descriptive words (e.g. comfortable, beautiful, soft) related to user perception of product. The second one concerns product descriptive words (e.g. color, material, shape). The third one concerns industrial process descriptors (e.g. process time for injection molding, or material for applying die casting process).

The SKIPPI system should be provided by proper words to each data bases and also the relations between these data bases in order to integrate both product designer and the engineering designer's view points to support the early stage of design process.

\section{KANSEI ENGINEERING}

Kansei Engineering was founded in Hiroshima University around 1970 with the works of Nagamachi (1). Kansei is a Japanese word which deals with sensitivity and aesthetics and includes customer's feeling about the product design, size, color, mechanical function, feasibility of operation and also the price. Kansei engineering is the first technique that uses customer's feeling as input and tries to find the relationship between the customer's feeling and the product features. For further information see also $(2,3)$.

\section{PRODUCT MODEL}

Product model is a common key-word in many researches which aim to structure knowledge and information related to the product. There are many approaches for product modeling.

FBS model of Gero (4) presents an ontology for design activity by definition of Function, Behavior and Structure domains and also the relations between the domains. Other models such as FBS-PPRE (5) and Core Product Model (CPM) were proposed to improve the FBS model. The CPM model (6) takes into account the geometry, the function, the material behavior, the 
functional and structural decomposition and the relations between these concepts in order to model the product. MOKA (7) uses the multi-view notion for product modeling. It considers the structural, functional, behavioral and technological views. The PPO (Product, Process, Organization) (8) model of IPPOP project, goes further by adding organization of system (collaboration view) to the product model. The multi-view product model (9) used in CoDeMo (Cooperative Design Modeler,12) is another product model that, by considering several view points up to design actors expertise, aims to support the integrated design methodology. All the mentioned models carry on the three main concepts of Function, Behavior and Structure and provide a valuable product representation. One of differences appears in the final objective of the model and also the representation language.

The Multiple-view feature modeling of Bronsvoort (10) supports the integral product development by providing an own view on a product for each design phase, and integrating all views. Each view contains a feature model of the product specific for the corresponding phase (conceptual design, assembly design, part detail design and part manufacturing planning). The model tries to connect conceptual features to assembly and manufacturing features. The Multiple-view feature modeling approach seems to be more relevant, in comparison to other mentioned models, for the integration of information to the early product development phase such as conceptual design. However the multiple-view feature model, like all the other studied product models, does not support the phase before conceptual design where our research problematic is positioned.

Nevertheless the multi-view product model of CoDeMo has also the same limitation, our work is based on this model.

\section{MULTI-VIEW PRODUCT MODEL}

The multi-view product model consists of both data model and knowledge model (9).The data model structures the skeleton of the product and enables the representation of the product through the different views of each participant. It defines the combination of three concepts; components, links and relations and indicates how interaction between these elements is possible. Figure 3 for example shows a graphical representation of components, links (characteristics) and relations. In this figure the structural decomposition of component 1 to sub components (comp1.1 and comp1.2) is illustrated. Each component has characteristics such as material, color, surface, dimension etc. Characteristics are called "links" in the Multi-View model. Relations can be built between two or more characteristics (links). The contact surface of two components for example, causes a relation between the characteristic "surface" of the two components.

The knowledge model applies features and production rules to capitalize knowledge of different trades, and to manipulate such knowledge into design process. Figure 4 shows the elements of data model and the elements of knowledge model which consists of factual knowledge (which deals with features) and temporal knowledge (which deals with production rules).

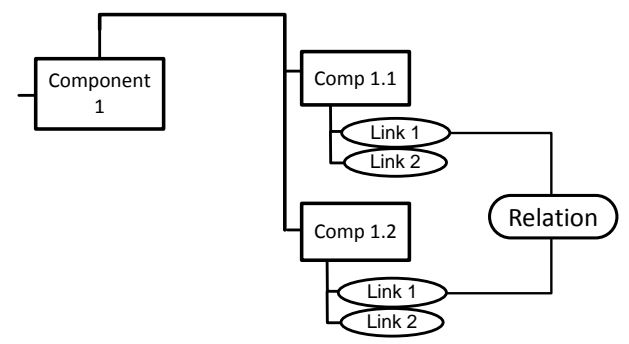

Figure 3: Graphical symbols of data models in Multi-View product model

\begin{tabular}{|c|c|c|c|}
\hline Elements of Data Model & Elements of Knowledge Model & \multicolumn{2}{|c|}{ Example: Manufacturing } \\
\hline Component: & Factual Knowledge & Plan: P1 & \\
\hline & - Constraint features & & Phase: ph1 \\
\hline Link & TemnoralKnowledar & $\begin{array}{l}\text { Feature } \\
\text { Plan }\end{array}$ & 5 \\
\hline & If premise then conclusion & $\begin{array}{l}- \text { time } \\
-\ldots\end{array}$ & Phase: ph2 \\
\hline Relation: & & $\begin{array}{l}\text { Phase } \\
- \text { n_opr } \\
-\ldots\end{array}$ & \\
\hline
\end{tabular}

Figure4: Product model associated between data model and knowledge model

Keeping the principles of the multi-view product model, we use a version of this product model which is adapted to our skippi context. The adapted model (which we call PP model) includes several views of the product; the structural view (the components, sub-components with the properties (or characteristics) such as weight, dimension, surface, material etc.; the functional view (the product functions, sub functions); the assembly view (the assembly solutions/operations, characteristics such as time of operation, cost, etc); the manufacturing view (the fabrication operation, characteristics such as time, cost, allowable tolerance and precision etc.) and the environmental view (recyclability, dust and vapor generation, energy consumption, etc.). Figure5 shows an illustration of the model with the 5 views and the characteristics attached to each component. The model includes also relations between the properties of components in one particular view or between different views. The relations are excluded from the figure 5 for better readability. 


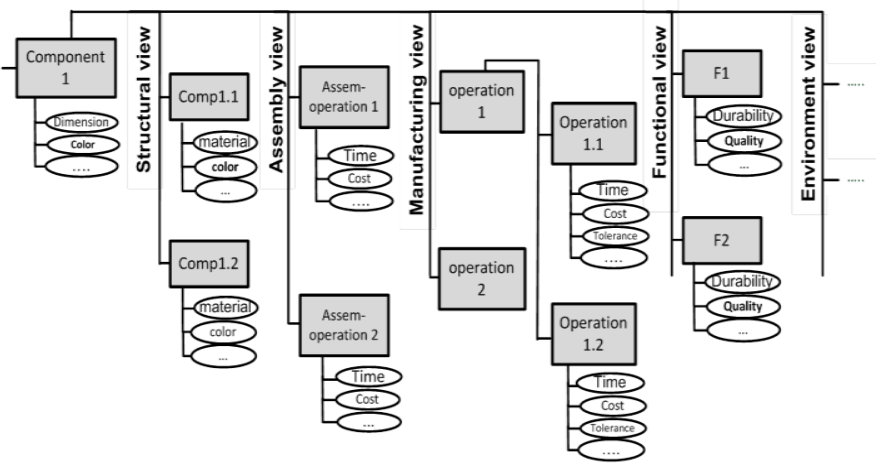

Figure5: Graphical representation of Product/Process (PP) multi-view model

Our research questions are then:

- Is this model capable to support the engineering designer's view point by providing an appropriate product representation of the brand product sector?

- Is the model capable to structure a way to enrich the SKIPPI system?

\section{THE RESEARCH METHOD}

In order to reply to the mentioned questions, some experimentations were performed with the participation of industrial partners of the SKIPPI project; "Mobiwire" a professional French company in development of communications products (such as cellphones) on collaboration with famous brands like Diesel, Swarovski and PUMA, and "Dièdre Design" which is an industrial design agency which offers product design solutions and services to the clients and collaborates with brands as well.

Our experimentation was carried out through interviews with some designers/engineers of Mobiwire and Dièdre Design companies and also through the collect of document specifications about the product itself and the realization process. We decided to focus on particular products to make concrete analysis of the structure and the realization processes of the products and then extend the initial results to a general analysis and classification of the related information about any product.

The data collection was organized in three phases: 1.Before interview; the companies were asked to prepare some documents such as the product specifications, product functions development, product assembly plan, manufacturing information and so on. A questionnaire was also prepared for interview sessions regarding to required information for Multiview product model. 2. During the interviews; the participants were asked to base their discussions around particular products (PUMA cellphone and Technifibre Tennis bag). The purpose was not the evaluation of Multi-view product model or comparing the Multi-view product model to the current product representation in the companies. The purpose was to gather data about descriptive product/process information. The questionnaire was used to guide the session. Both sessions were audio recorded. 3. After interview; an initial data classification was realized from gathered documents, and audio files. The product samples were then analyzed and -in the cellphone casedisassembled to get a better vision of the structure. All the information was then arranged in an Excel file for each product. The table contains the components list, with an image and a reference number for each component. Other data, concerning the component's characteristics (color, material, shape and so on), component function(s), manufacturing process, and assembly position and process of the component with other components is also included in the table. Figure 6 shows selected parts of each mentioned table.

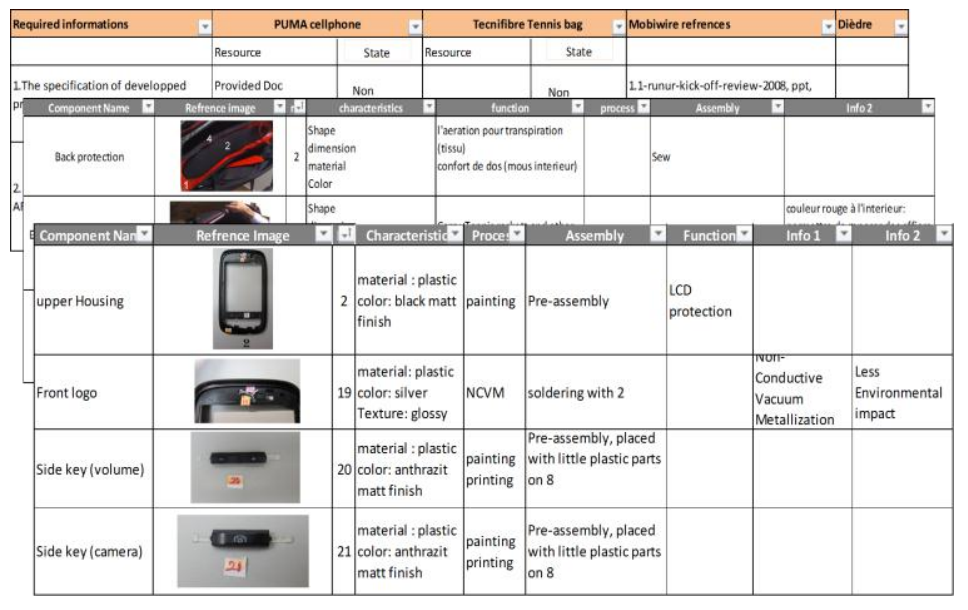

Figure 6: Initial information analyze and classification of the experimentation

In parallel to this study, another team (LCPI, product design and innovation laboratory from Paris Art et Métier University) was working on design issue considering Kansei engineering, semantic and emotional perception of product and the interpretation of brand values by interviewing the mentioned companies. Three experimentations were performed (Figure 7). Since both Mobiwire and Dièdre Design did not have formalized design process and did not use particular information system, the objective of the first experimentation was to understand Mobiwire and Didère Design activities in general, in order to bring out sharing points and common specificities of the companies. The second experimentation aimed at going more precisely through the descriptions of data or information manipulated by companies' product designers to formalize a general structure of Kanesi/Product/Process corpus. Initial categories were resulted from this experiment by designers' posterior verbalizations about realized projects. The objective of the third experimentation was to underline the relations that product designer makes between these categories, or relations between data in the same category (13). Therefore the study of the LCPI team was less focused on one particular product and was rather covering the overall product sectors of the companies. 


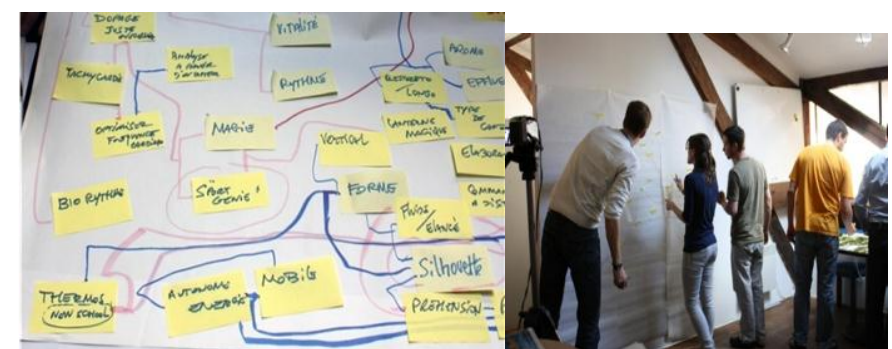

Figure 7: Data categorization through LCPI experimentations

\section{CASE STUDY}

For the purpose of building the adapted Multi-View model, two particular products were chosen. The first one was a cellphone produced by Mobiwire for the sport brand PUMA. The second product was a sport bag designed by Dièdre for the brand "Tecnifibre". The case studies are special since both of them consider highly the visual design aspect of product in order to fulfill the brand's representation and satisfaction. One of the companies (Mobiwire) has both engineer and design culture, while the other one (Dièdre) was rather design oriented.

The data model was accomplished for the PUMA cellphone and the Tennis bag. In the fallowing paragraphs we explain an extract of data model for PUMA cellphone.

For a particular component (Front Logo) of PUMA cellphone, a part of the data model is illustrated in figure 8. Decomposition of a component allows different level of abstraction in structural view. The upper level gives a view of the system in its totality. Decreasing the level with decomposition enables the understanding of how the product is made and also allows reaching detailed specification of parts. The component is represented schematically by a rectangle, with a name and a reference number. The Front Logo 19 is a part of Upper Housing which it-self is a part of Body. Some characteristics of Front Logo are the plastic material and the silver color. Such characteristics which allow an external consideration of the component, are the "Links" in Product model (9). Links (or characteristics) are represented by oval and is hung below the specified component. A side from the structural view, the Assembly and Manufacturing views provide a list of operations related to all sub-components of the upper component into which these views are associated. The Front Logo19 is assembled to Upper Housing2 by soldering operation. The unrefined component is obtained by Injection molding and then through the NCVM (Non Conductive Vacuum Metallization) operation, a thin nonconductive metal film is formed on the molded plastic surface.

The model tends to represent on one hand the product and process properties (PP) and on the other hand the product/process relationships (PP relationships). However the relations are excluded from figure 8 to simplify the scheme. A relation may be specified between two or several links (characteristics). Relation may exist between the links of the same component, between the links of different components of the same view or different views.

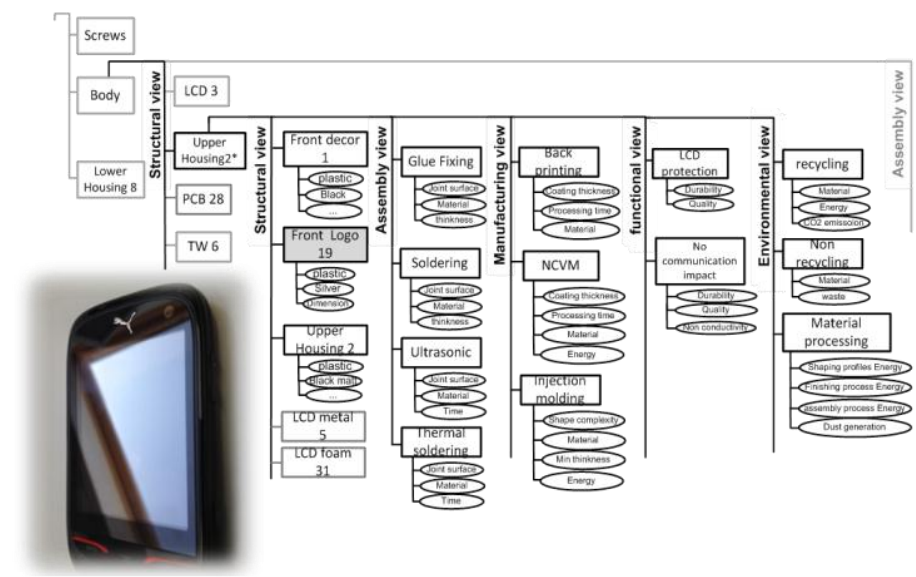

Figure 8: Selected part of PP model for Front Logo component of PUMA cellphone

As each view is a particular decomposition of a component, the relations at this level concern the necessity or the constraints between characteristics of components. The relations are intermediate elements which do not appear in the final description of the product while they play an important role in the effects of eventually change of any component, on other components or on the whole product structure. Building a relation between two characteristics is the result of expert knowledge reasoning. For the Front Logo, some of the relations (shown in Figure 9) are as follows: There is a relation between the plastic material of this component, and the possible materials for molding operation (R1). Also the shape of Logo is compatible with the range of shape complexity that could be realized by the molding operation (R2). The Soldering assembly of the Logo on the Upper housing is possible because the contact surface between the Logo and the Upper housing is compatible with the required joint surface of the soldering operation (R3). The Silver color of Logo is included in the color range which is supported by NCVM process (R4). The special metal film used in NCVM process, prevents radio interference and doesn't require any measures against electrostatics, so that it participates in the "No communication impact" Function (R5).Applying the NCVM process causes less environmental load than other alternative finishing processes (R6). 


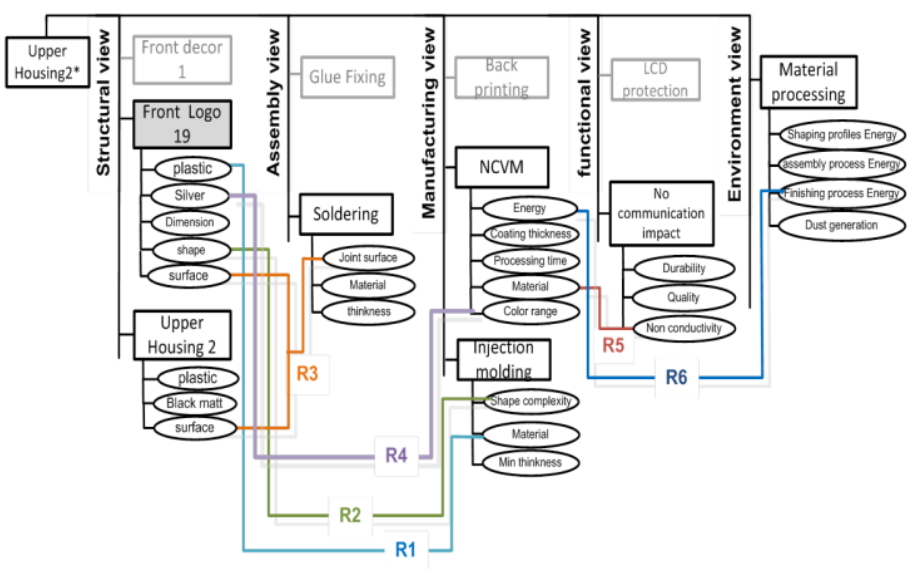

Figure 9: Example of graphical relation visualization in PP model

\section{RESULTS}

Two main results were obtained through this study, which reply to our earlier research questions. The first one is about the use of the PP model in the field of studied products and in the context of the SKIPPI project. The second one concerns the relevance of such a product model to structure a way to enrich the SKIPPI system in coupling the product designer's model to engineer's model.

\section{An appropriate product representation to support engineering designer's view}

The Multi-View product model used in CoDeMo software indeed tends to support the collaborative work of several actors during the design process. Therefore it considers the knowledge model of experts and the data model to provide a collaborative work space aiming to integrate the manufacturing knowledge as soon as possible in the design process of rather mechanical products. The model was not been tested for brand products for which the appearance and visual design of the product is highly considerable. In this study, using the principles of the Multiview product model, we built a Product/Process (PP) model not as a collaboration support tool during the product design but as a tool to analyze manufactured brand products. The main objective of the PP Model is to structure the engineering knowledge about product and process descriptions and to provide appropriate data to be integrated in the upstream phase of product designing. Definition of several views such as structural, assembly, manufacturing and functional views (which are already a part of Multi-View model) and the environmental view (which we added to PP model), is done according to this objective. The structural view gives an overall view of the component's positions in a hierarchical order and thus provides the product structure representation. The assembly and manufacturing views provide processing information about associated components. The functional view provides information about functions development (service function and technical functions). The environmental view gives some elements of evaluation of components and applied processes in regard to environmental impacts.

We built the PP model for two brand products from very different sectors, a cellphone (mechanic and electronic sector) and a sport bag (textile sector) as it was described in previous section. The first result is that the PP model covers the product representation through different views and gives the possibility to find relations between product and process parameters. Therefore the PP model is relevant to analyze the structure of developed products according to engineering designer's view.

Nevertheless we had to make some choices associating some elements in the PP model. Some exterior elements which are used to realize a process operation, such as "Glue" or "Screw" could also be considered as a structure component for the product. In these cases we decided to consider the outside visibility of the element as the criteria for category association. Therefore the screw was kept as a product component in structural view as it appears in product design and affects the perception aspects, while the "Glue" was considered only as the criteria for "Glue fixing" operation in assembly view of PP model. The same difficulty came up with "zip" and "sewing" operation in tennis bag. "Zip" was kept as structural component while "sewing" operation should be placed in structural category when it has decorative visualization. Otherwise depending on the precision level, sewing can be seen as an assembly or a manufacturing process.

Providing adequate information for the SKIPPI system:, Three main categories which are defined during the LCPI experimentations contain Kansei category, Product category and Process category, and each of them contains sub-categories. Some examples of sub-categories are Kansei (value, style, gesture, emotions, brand), Product (functionality, form, motif, color, texture, material, component, sector) and Process.

Even if the PP model deals with concrete information about the product and process, and classifies the information in a hierarchical structure order, the model still provides adequate information for the SKIPPI system.

Instead of giving specified structure, the PP model helps to classify engineering information in several categories. For example the PP model provides the Process category of SKIPPI with sub-categories (manufacturing, assembly, and environmental impact). The model also rearranges the subcategories of SKIPPIs' Product category by defining "component" as a sub-category with characteristics such as "form, motif, color , material" and the "function" as another sub-category with expected performances (such as durability, quality, etc.).

Furthermore, connections between the categories are provided by the PP model through the relations between the characteristics of two components, or a characteristic of a component and a characteristic of a process. Hence the second result is that the PP model is capable of enriching the SKIPPI 
system by generating categories and the relations between the categories.

Going back to the initial SKIPPI illustration of the Kansei/Product/Process connection (Figure 2), our experiment showed that there are two product representations, one of them considers the overall product aspect that can be connected to Kansei domain, and the other one considers the technical and detailed aspects of product that can be connected to industrial processes (Figure 10).

One of the differences appears with the "component". The "component" is defined as a product sub-category to which characteristics such as "form, motif, color, and material" are associated (as we did in our product representation). In the other representation, the "component" is considered as a characteristic, as well as "color, form and material" for the overall product (result of LCPI works).

It could be said that from the product designer's view, the product representation exists according to the product designer's interpretation and description of the product. The representation concerns Kansei science (semantics, emotion and values translation to product elements), and Kansei/Product relations. We call such a representation the KP model. To couple engineering designer's model (PP) to design model (KP), the KPP model of SKIPPI should take into account the Product/Process attributes and relations from one side, and the Kansei/Product attributes and relations from the other side and connects them together (Figure 10).

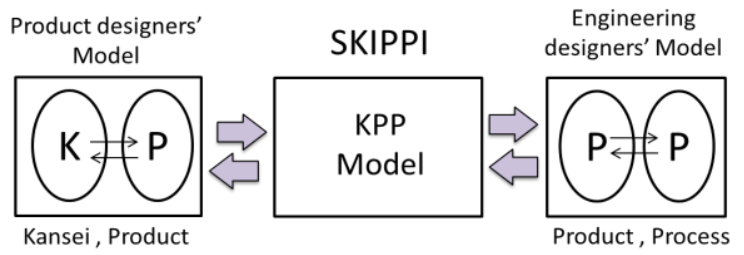

Figure 10: KPP Model of SKIPPI should consider both engineering designer and product designer models

The first step is taken in this study by showing the potential of PP model which carries the contribution of engineering designer's view to the KPP model. The KPP model on the other hand, by considering the product designer's model, can enrich the PP model in order to make the PP model more compatible with KP model.The next step in SKIPPI project is to connect engineering designer's representation to the designer's representation through a software. This first software prototype would be then evaluated and evolved.

\section{CONCLUSION}

This paper deals with the PP product model and aims to illustrate the connection between engineering knowledge and product design knowledge through the SKIPPI system.

The product/process multi-view model supports engineering designers' view through definition of several views on product. It allows not only the structuring and tracing of the relative knowledge of the engineering designers on the product but also permits the creation of relationships with the product designer's representation through the lexical categories. The more compatible the engineer's product model with designer's product model is, the easier the sharing of their technical views and their collaboration will be.

Other experiments are actually taking place with the project partners to find out the possible dialoging way between the KP and PP models.

\section{REFERENCES}

1) Nagamashi M., Kansei Engineering as a powerful consumer-oriented technology for product development, Applied ergonomics, 2002, Vol 33, 289-294.

2) Hsiao, Shih-Wen, Fu-Yuan Chiu, and CS Chen, Applying aesthetics measurement to product design. International Journal of Industrial Ergonomics 38, no. 11-12 (November 2008): 910-920.

3) Nagamachi M., Kansei Engineering: A new ergonomic consumer-oriented technology for product development. International Journal of Industrial Ergonomics 15, no. 1 (January 1995): 3-11.

4) Gero J.S., The situated Function-Behaviour-Structure Framework, Artificial Intelligence in Design, 2002, 89-104

5) Gonçalves M., Formalization of transfers of project specifications in the design cycle of manufactured product (French title: Formalisation des transferts de spécifications projet dans le cycle de conception de produit manufactures : application à un environnement de type Produit-ProcessusOrganisation), PhD Thesis, Bordeaux University 1, 2010

6) Fenves J.S. A core product model for representing design information, Technical report, National Institute of Standards and Technology (NIST), 2002

7) Brimble B. et al. A Guide to Knowledge-Based Engineering: The MOKA Methodology

8) Noël F, Roucoules L., The PPO design model with respect to digital entreprise technologies among product life cycle, International Journal of computer Integrated Manufacturing, vol21, No2, 2008, 139-145

9) Tichkiewitch S., Veron M., Methodology and product model for integrated design using a multiview system, Annals of CIRP, 1997, 46(1)

10) Bronsvoort WF., Multiple-view feature modeling for integral product development, computer-aided design 36(10), 2004, 929-946

11) Pahl G., Beitz W., Engineering Design, A systematic approach, 1996, Springer, London

12) Roucoules L. Method and knowledge to assist the development of an integrated design environment. (French title: Méthodes et connaissances : contribution au développement d'un environnement de conception intégrée). PhD Thesis. INP Grenoble, 1999

13) SKIPPI, Requirement analysis report, 2011, http://www.skippiproject.com/ 